\title{
Crescimento de tangerinas 'Ponkan' em plantas submetidas ao raleio químico ${ }^{1}$
}

Maria do Céu Monteiro da Cruz², José Darlan Ramos ${ }^{3}$, Rodrigo Amato Moreira ${ }^{4}$,Verônica Andrade dos Santos ${ }^{5}$

\section{RESUMO}

Pesquisas comprovam que a relação entre o número de frutos e seu tamanho influencia o rendimento da produção e a qualidade dos frutos. Este trabalho foi conduzido com o objetivo de avaliar o desenvolvimento de tangerinas 'Ponkan', produzidas em plantas enxertadas sobre limoeiro ‘Cravo', submetidas ao raleio químico com a aplicação de ethephon. As plantas foram pulverizadas com cinco concentrações de ethephon (0, 150, 300, 450, $\left.600 \mathrm{mg} \mathrm{L}^{-1}\right)$, aplicadas em dois estádios de desenvolvimento dos frutos (30 e 40 mm de diâmetro transversal). Em cada planta, foram selecionados quatro ramos, localizados nos quatro quadrantes, com apenas uma fruta, para avaliar seu desenvolvimento por meio do diâmetro transversal. O raleio químico com a aplicação de ethephon favoreceu a obtenção de tangerinas com maior tamanho. As concentrações a partir de $300 \mathrm{mg} \mathrm{L}^{-1}$ de ethephon foram as mais adequadas para promover o raleio da tangerina 'Ponkan', nas condições estudadas. O raleio realizado no estádio de 40 mm proporcionou a obtenção de frutos de maiores diâmetros.

Palavras-chave: Citrus reticulata Blanco, tamanho de fruto, fitorreguladores, ethephon, manejo.

\section{ABSTRACT}

\section{Fruit growth of 'Ponkan' mandarin in trees subjected to chemical thinning}

Research has shown that the relationship between fruit number and fruit size influences yield and fruit quality. The aim of this work was to evaluate mandarin 'Ponkan' fruits' growth when submitted chemical thinning with the application of ethephon. The plants were sprayed with five ethephon concentrations (0, 150, 300, 450 and $\left.600 \mathrm{mg} \mathrm{L}^{-1}\right)$, at two fruit development stages (30 e $40 \mathrm{~mm}$ of cross diameter). Four shoots in each quadrant with a single fruit were chosen per plant. In this fruit, growth was to evaluate through the cross diameter. Production of larger fruits was favored by chemical thinning with ethephon application. Ethephon concentrations above $300 \mathrm{mg} \mathrm{L}^{-1}$ were more effective for thinning of 'Ponkan' mandarin under the conditions studied. Thinning carried out at the 40-mm stage provided fruits of higher diameter.

Key words: Citrus reticulata Blanco, ethephon, fruit size, management, phytoregulators.

Recebido para publicação em outubro de 2008 e aprovado em outubro de 2009

${ }^{1}$ Parte da tese de doutorado em Fitotecnia da primeira autora pela UFLA. Apoio: CNPq

${ }^{2}$ Engenheira-Agrônoma, Doutora. Universidade Federal dos Vales do Jequitinhonha e Mucuri, Faculdade de Ciências Agrárias/Agronomia, Rodovia MGT 367, Km 583, nº 5.000, 39100-000, Diamantina, Minas Gerais (MG), Brasil. m_mariceu@yahoo.com.br

${ }^{3}$ Engenheiro-Agrônomo, Doutor. Departamento de Agricultura, Universidade Federal de Lavras (UFLA), Cx. Postal 3037, 37200-000, Lavras, MG. darlan@ufla.br

${ }^{4}$ Engenheiro-Agrônomo. Mestrando em Fitotecnia, UFLA, Cx. Postal 3037, 37200-000, Lavras, MG. amatomoreira@yahoo.com.br

${ }^{5}$ Engenheira-Agrônoma, Mestre. Doutoranda em Fitotecnia, UFLA, Cx. Postal 3037, 37200-000, Lavras, MG. 


\section{INTRODUÇÃO}

O desenvolvimento do fruto e o seu tamanho final são fundamentais para a qualidade e o rendimento da produção da tangerineira 'Ponkan', quando os frutos se destinam ao mercado para consumo "in natura”.

O tamanho dos frutos é influenciado diretamente pelo número de frutos na planta. De acordo García-Luis et al. (2002), a massa individual de frutos até a maturidade é inversamente relacionada com a quantidade de frutos por planta, em decorrência da competição entre os drenos, que são os frutos e as folhas jovens.

O tamanho depende da relação fonte-dreno, a qual é responsável pela quantidade de fotoassimilados que é distribuída para cada fruto (Guardiola \& García-Luis, 2000). As informações sobre o efeito da relação fonte-dreno permitem a compreensão das interações fisiológicas que influenciam o desenvolvimento de frutos e a produção (Minchin et al., 1997).

O fator limitante para o crescimento de frutos varia de acordo com o seu estádio de desenvolvimento. O crescimento dos frutos cítricos é do tipo sigmoidal simples, que é dividido em três fases. A fase de crescimento inicial é caracterizada pelo aumento da espessura do pericarpo, resultante da divisão celular; a segunda fase é caracterizada pelo rápido crescimento, em decorrência do aumento do volume celular e dos lóculos e da redução da espessura do pericarpo; na terceira fase ocorrem a pigmentação da casca e a maturação, com aumento do conteúdo de sólidos solúveis e redução da acidez (Bain, 1958).

Dependendo do estádio de desenvolvimento dos frutos, os carboidratos se correlacionam de forma positiva com o crescimento, pois os frutos são considerados drenos de utilização no período de divisão celular (fase I do crescimento) e, durante o estádio de expansão celular (fase II do crescimento), atuam como drenos de armazenamento (Mehouachi et al., 1995). Por esta razão, o estádio de desenvolvimento do fruto no momento do raleio tem recebido maior atenção, visto que o seu crescimento até a colheita é dependente, além das condições ambientais, dos fatores endógenos relacionados com a abscisão.

Para aumentar o tamanho de frutos, várias práticas têm sido realizadas, entre elas, o raleio químico (Zaragoza et al., 1992). Esta prática aumenta a disponibilidade de carboidratos e reduz a competição entre os drenos e favorece melhoria no tamanho dos frutos (Spiegel-Roy \& Goldschmidt, 1996).

Dentre os produtos que podem ser utilizados no raleio químico, o ethephon, um fitorregulador que libera etileno, em contato com o tecido vegetal, promovendo a abscisão (Sanches, 2000), tem sido aplicado nas espécies frutífe- ras, em concentrações que variam de 150 a $400 \mathrm{mg} \mathrm{L}^{-1}$, apresentando percentuais de raleio variáveis em função da época de aplicação. Além do etileno, auxinas, giberelinas (Talón et al., 1990) e ácido abscísico (Agustí et al., 2007) podem agir como intermediários no processo de abscisão em citros.

O raleio realizado com a finalidade de aumentar o tamanho dos frutos deve ser feito quando eles estão com o diâmetro transversal medindo entre 15 a $20 \mathrm{~mm}$ (Ortolá et al., 1998), ou logo após o final do período de queda fisiológica dos frutos, quando se encerra a fase de divisão celular (Agustí et al., 1998). O raleio tardio pode afetar o crescimento dos frutos, porque ocorre em um momento em que as reservas de carboidratos na planta já foram transportadas e utilizadas pelos frutos (Ruiz et al., 2001).

O controle do número e do tamanho final de frutos tem despertado o interesse dos produtores, em função da exigência do mercado, que prefere frutos de tamanhos maiores, pois nos anos de alta produção, os frutos são pequenos e de baixa qualidade, o que dificulta sua comercialização.

O presente trabalho foi realizado com o objetivo de avaliar o efeito do raleio químico, com a aplicação de ethephon, sobre o desenvolvimento de tangerinas 'Ponkan'.

\section{MATERIAL E MÉTODOS}

O experimento foi conduzido durante o período de janeiro a julho de 2007, em pomar comercial no município de Perdões, Sul de Minas Gerais. A altitude média da região é de 900 metros e o tipo climático é Cwb, segundo a classificação de Köppen, caracterizado com verões quentes e úmidos e invernos secos e frios. Durante o período de avaliação, foram registrados os dados de temperatura, precipitação e umidade (Figura 1). O solo é classificado como Argissolo Amarelo distrófico típico (Empresa Brasileira de Pesquisa Agropecuária - Embrapa, 2006).

As avaliações foram realizadas em frutos de tangerineira ‘Ponkan' (Citrus reticulata Blanco cv. Ponkan), enxertada sobre limoeiro ‘Cravo' (C. limonia Osbeck). As plantas estavam com dez anos de idade, conduzidas sem irrigação, no espaçamento de $6 \mathrm{~m}$ x $3 \mathrm{~m}$. As adubações foram realizadas de acordo com análises foliares e de solo, parceladas em quatro aplicações, e, os tratos culturais, conforme as recomendações técnicas para a cultura.

Utilizou-se o esquema fatorial 5 x 2 (concentrações de ethephon x estádio de desenvolvimento do fruto), disposto em blocos casualizados, com quatro repetições e sete plantas por parcela, com avaliações realizadas nas três centrais. Os fatores foram as concentrações de ethephon: 0, 150, 300, 450 e $600 \mathrm{mg} \mathrm{L}^{-1}$ e os dois estádios 


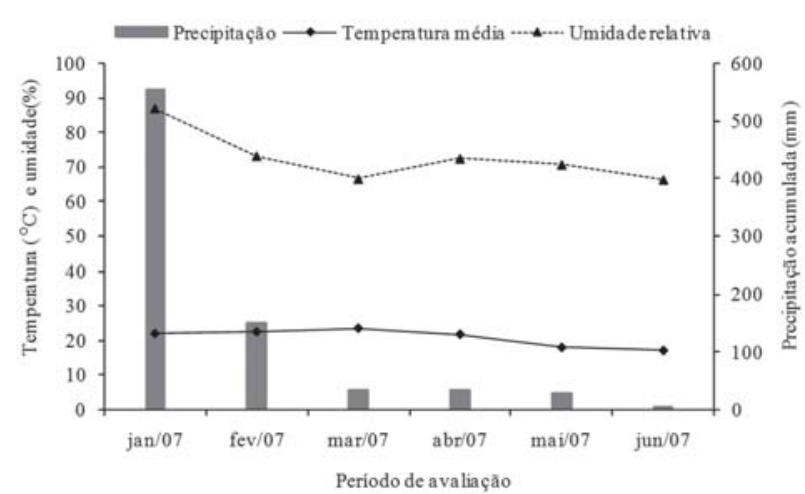

Figura 1. Médias mensais da temperatura, umidade relativa e precipitação, de janeiro a julho de 2007, no município de Perdões, Minas Gerais.

(Fonte: Estação de meteorológica do Departamento de Engenharia da UFLA, Lavras, MG, 2009).

de desenvolvimento dos frutos: 30 e 40 mm de diâmetro transversal, com parcelas subdividas no tempo, referente às avaliações realizadas durante a fase de crescimento dos frutos.

Antes da aplicação dos tratamentos, as plantas foram selecionadas quanto ao seu potencial produtivo, de forma que todas as plantas submetidas aos tratamentos apresentassem carga expressiva de frutos. Além disso, foram realizadas amostragens semanais para determinar os estádios de desenvolvimento dos frutos no período da aplicação.

Em janeiro de 2007, as plantas foram pulverizadas em toda a extensão da copa (interna e externa), com ethephon, utilizando-se o produto comercial ZAZ ${ }^{\circledR}$, concentrado solúvel contendo $480 \mathrm{~g} \mathrm{~L}^{-1}$ do ácido 2-cloroetil fosfônico.

Para a aplicação do ethephon, foi utilizado um pulverizador costal com pressão de $6 \mathrm{kgf} \mathrm{cm}^{-2}$, de bico cônico com capacidade de deposição de partículas em torno de 70 a 100 gotas $\mathrm{cm}^{-2}$, com diâmetros de 100 a 200 micra, obtendo-se molhamento homogêneo de toda a cobertura foliar.

Foram aplicados, aproximadamente, $2 \mathrm{~L}_{\text {planta }}{ }^{-1}$, volume este determinado, anteriormente, mediante teste em branco com a aplicação de água, para molhar toda a copa de maneira uniforme e evitar a deriva do produto.

Em cada planta foram selecionados quatro ramos, localizados nos quatro quadrantes (norte, sul, leste e oeste), contendo apenas um fruto por ramo, com diâmetro em torno de $30 \mathrm{~mm}( \pm 0,05 \mathrm{~mm})$ e $40 \mathrm{~mm}( \pm 0,05 \mathrm{~mm})$, determinado a partir de amostragens feitas em todas as plantas de cada tratamento, o que correspondeu a 240 frutos para cada estádio.

Após a pulverização das plantas, o número de frutos removidos por planta pela ação da aplicação do ethephon foi avaliado, aos 3, 6, 9 e 12 dias. Foi avaliado, semanalmente, nos ramos marcados, o diâmetro transversal dos frutos até o início da maturação, que ocorreu em junho de
2007, quando os frutos apresentaram coloração alaranjada. Na colheita, foram determinados o número de frutos colhidos, a massa fresca e os diâmetros, transversal e longitudinal, dos frutos.

Os dados obtidos foram submetidos à análise de variância e de regressão, por meio do software SAS. Optou-se pelo desdobramento de todas as interações, no sentido de estudar o comportamento do desenvolvimento do fruto, em função das épocas avaliadas e das concentrações aplicadas em ambos os estádios de desenvolvimento.

Para a escolha dos modelos foram considerados a dispersão dos resíduos, o potencial para explicar o fenômeno biológico em questão e os testes de significância das variáveis e do coeficiente de regressão, utilizando-se o teste t a $5 \%$ de probabilidade de erro.

\section{RESULTADOS E DISCUSSÃO}

Houve interação significativa entre as concentrações de ethephon, os estádios de desenvolvimento dos frutos no momento da pulverização e as épocas de avaliação do desenvolvimento dos frutos, avaliados por meio do diâmetro transversal. Foi observado efeito do ethephon sobre o número de frutos raleados e colhidos por planta, a massa fresca e diâmetros transversal e longitudinal dos frutos, avaliados na época da colheita.

Quando foram ajustados os dados em função de épocas e de concentrações de ethephon aplicadas, obtiveram-se equações para superfícies de resposta semelhantes nos dois estádios de desenvolvimento (Tabela 1). O maior desenvolvimento no diâmetro transversal dos frutos foi estimado, a partir das equações obtidas, nas plantas pulverizadas com a concentração de $300 \mathrm{mg} \mathrm{L}^{-1}$, aos 61 dias após a realização do raleio, representado pelos diâmetros de 57,6 e 63,4 mm para os estádios de $30 \mathrm{~mm}$ e 40 $\mathrm{mm}$, respectivamente.

O maior desenvolvimento no diâmetro dos frutos observado aos 61 dias após a aplicação do ethephon pode ser atribuído ao aumento do volume celular e preenchimento dos lóculos por fotoassimilados, durante o estádio de armazenamento, que confere a expansão do fruto (Mehouachi et al., 1995).

Quanto ao maior desenvolvimento dos frutos favorecido pela aplicação do ethephon para promover o raleio, possivelmente, ocorreu em função da maior da disponibilidade de carboidratos para os frutos remanescentes (Spiegel-Roy \& Goldschmidt, 1996). No entanto, comparando-se o diâmetro, associado às épocas de avaliação, verificou-se que a concentração de $300 \mathrm{mg} \mathrm{L}^{-1}$ favoreceu o maior desenvolvimento, o que pode ter ocorrido em função do estresse sofrido pelas plantas pulverizadas com as concentrações maiores, em decorrência da queda de 
Tabela 1. Equações ajustadas para o desenvolvimento do diâmetro transversal em tangerina 'Ponkan', em função das concentrações de ethephon e de épocas (dias de avaliação)

\begin{tabular}{llc}
\hline Estádio & \multicolumn{1}{c}{ Equação } & $\mathbf{R}^{2}$ \\
\hline $\mathbf{3 0} \mathbf{~ m m}$ & $\mathrm{Z}=23,792+0,021 \mathrm{x}+0,457 \mathrm{y}-0,00002 \mathrm{x} * \mathrm{x}+0,0002 \mathrm{x} * \mathrm{y}-0,001 \mathrm{y} * \mathrm{y}$ & 0,97 \\
$\mathbf{4 0} \mathbf{~ m m}$ & $\mathrm{Z}=33,068+0,024 \mathrm{x}+0,496 \mathrm{y}-0,00002 \mathrm{x} * \mathrm{x}+0,0001 \mathrm{x} * \mathrm{y}-0,002 \mathrm{y} * \mathrm{y}$ & 0,96 \\
\hline
\end{tabular}

$\mathrm{x}$ = concentrações e ethephon e $\mathrm{y}=$ época de avaliação

folhas maduras, que atuam como fontes de fotoassimilados, podendo limitar o seu fornecimento para os frutos logo após a pulverização das plantas.

Embora a queda de folhas, visualmente observada, não tenha comprometido o desenvolvimento final e a qualidade dos frutos (Cruz et al., 2009), inicialmente pode ser considerada um fator que tenha atrasado o seu desenvolvimento, pois, após a abscisão, ocorreu à emissão de algumas folhas novas, que também são drenos que competem com os frutos em desenvolvimento. Por isso, para a aplicação de concentrações maiores de ethephon para promover o raleio, além do estádio de desenvolvimento dos frutos, deve-se considerar a abscisão de folhas, que se ocorrer de forma intensa pode ser prejudicial ao desenvolvimento dos frutos remanescentes.

O maior diâmetro, observado nos frutos das plantas que foram submetidas ao raleio no estádio de $40 \mathrm{~mm}$, pode ser atribuído à maior capacidade de desenvolvimento dos frutos maiores, em decorrência da sua maior força de dreno, quando comparada à força de dreno dos frutos que caíram, em função do acúmulo de metabólitos provido pela maior disponibilidade de carboidratos e elementos minerais de suas inflorescências (Ruiz \& Guardiola, 1994).

Dessa forma, os frutos persistentes passaram a depender exclusivamente da disponibilidade de assimilados para expressarem seu potencial de crescimento. Isso por que, durante o estádio de expansão celular (fase II do crescimento), todos os frutos já passaram pela fase formação, ou seja, já ocorreu a divisão celular, que é a fase que determina o tamanho do fruto.

Apesar de a maioria dos trabalhos mencionarem que o raleio não deve ser promovido em estádios avançados de desenvolvimento dos frutos, em função do consumo de reservas já carreados para os frutos, o raleio no estádio mais avançado $(40 \mathrm{~mm})$ possibilitou a eliminação de frutos com menor potencial para se desenvolverem sob condições favoráveis ao seu crescimento.

O desenvolvimento do diâmetro transversal do fruto em função da época foi ajustado ao modelo exponencial, em ambos os estádios de desenvolvimento (30 e $40 \mathrm{~mm}$ ), para todas as concentrações testadas (Figuras 2A e 2B). Observou-se que houve aumento do diâmetro transversal ao longo do tempo. Porém, a magnitude do acréscimo variou de acordo com a concentração de ethephon e do estádio de desenvolvimento dos frutos, constatando-se que os melhores resultados foram obtidos no estádio de $40 \mathrm{~mm}$.

A diferença observada em relação ao tamanho do fruto nas plantas pulverizadas com ethephon ocorreu em função da redução no número de frutos, principal-

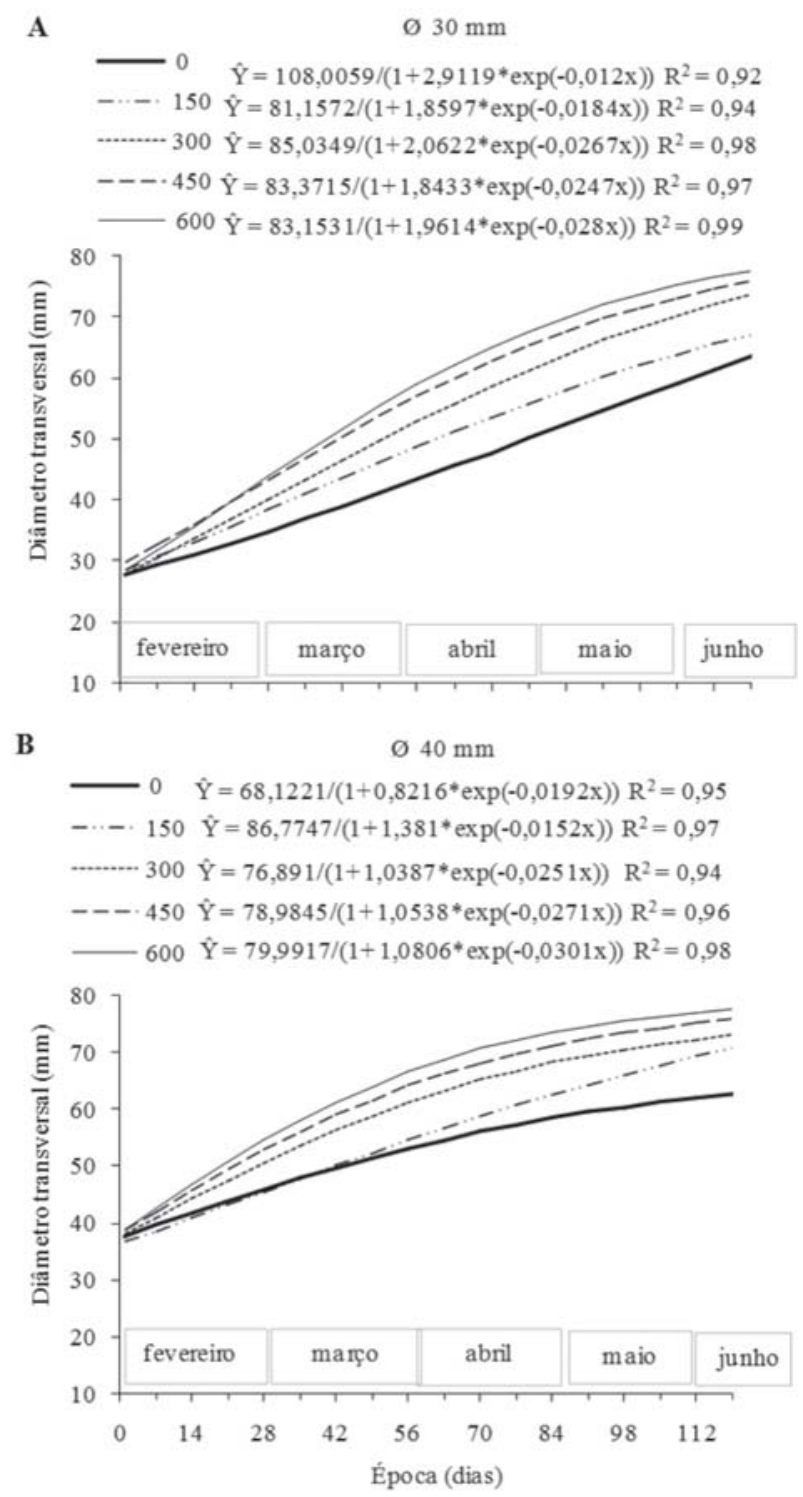

Figura 2. Diâmetro transversal de frutos de tangerineiras 'Ponkan' (Citrus reticulata Blanco), pulverizadas com as concentrações de ethephon (0, 150, 300, 450 e $\left.600 \mathrm{mg} \mathrm{L}^{-1}\right)$, aplicadas no estádio de $30 \mathrm{~mm}$ (A) e de $40 \mathrm{~mm}$ (B), ao longo do período de avaliação. 
mente, nas tangerineiras pulverizadas com as maiores concentrações (Figuras 3A e 3B). Isso por que a maior abscisão de frutos foi maior nas plantas pulverizadas com a maior concentração de ethephon, $600 \mathrm{mg} \mathrm{L}^{-1}$, independentemente do estádio de desenvolvimento do fruto no momento do raleio.

Essa relação entre o tamanho e o número de frutos por planta demonstra que o crescimento destes depende da relação fonte-dreno, a qual controla a disponibilidade de fotoassimilados para os frutos em desenvolvimento (Guardiola \& García- Luís, 2000).

O comportamento observado no desenvolvimento dos frutos em função do modelo ajustado está de acordo com o comportamento característico para o crescimento de frutos, que, apresentam aumento do volume celular e dos lóculos preenchidos pelas vesículas de suco, à medida que estes absorvem água. Este comportamento caracteriza a segunda fase do crescimento dos frutos cítricos, passando a ocorrer de forma lenta, quando se inicia a terceira fase do crescimento, marcada pela fase de maturação.

Comparando-se o desenvolvimento do diâmetro dos frutos, independentemente da época, observou-se com-
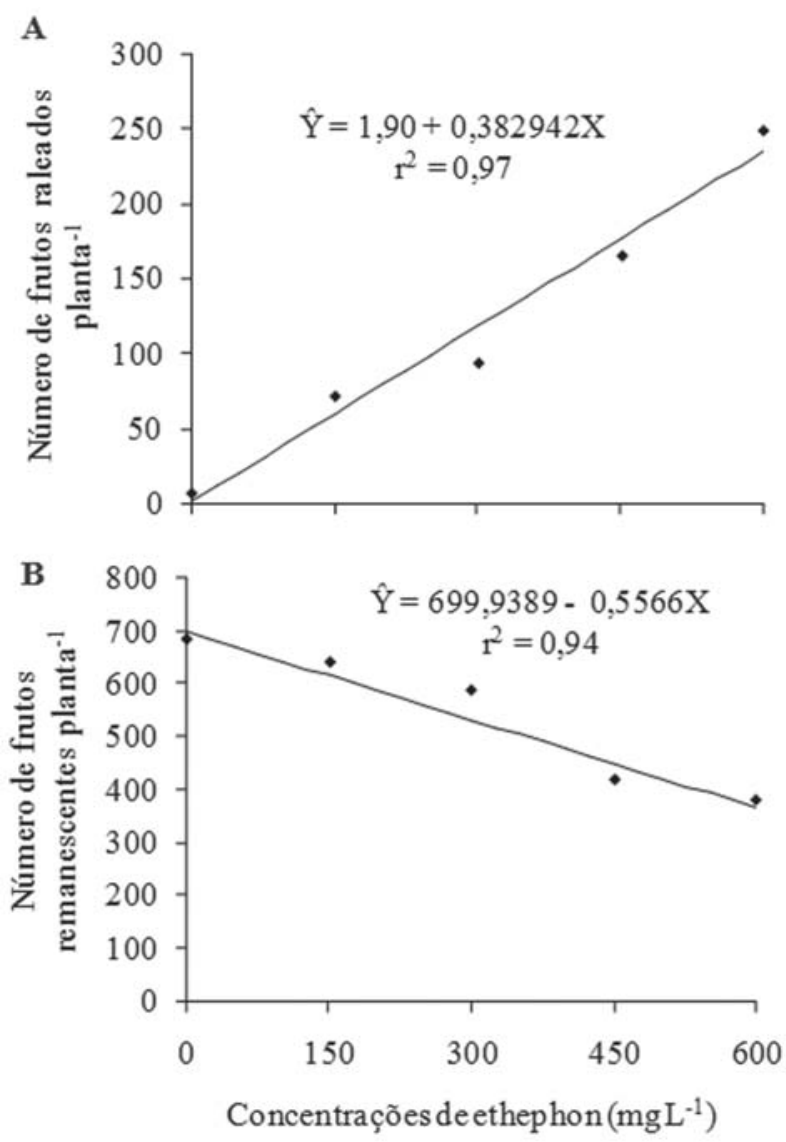

Figura 3. Número de frutos raleados (A) e colhidos (B) de tangerineira 'Ponkan' (Citrus reticulata Blanco), em função das concentrações de ethephon.

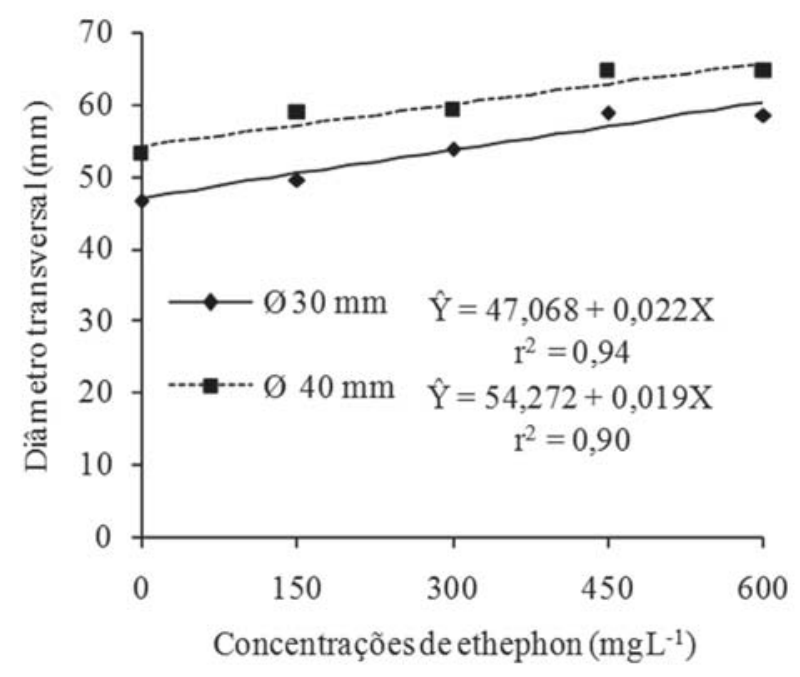

Figura 4. Diâmetro transversal de frutos de tangerineira 'Ponkan' (Citrus reticulata Blanco), em função das concentrações de ethephon nos estádios de 30 e $40 \mathrm{~mm}$.

portamento linear, na medida em que se aumentou a concentração de ethephon, em ambos os estádios de desenvolvimento (Figura 4). Comparando-se com as plantas que não foram pulverizadas, foi possível observar, naquelas pulverizadas com a concentração de $600 \mathrm{mg} \mathrm{L}^{-1}$, acréscimos no diâmetro dos frutos de 28,6\% e 21\%, para os estádios de $30 \mathrm{~mm}$ e $40 \mathrm{~mm}$, respectivamente. Esse resultado evidencia que o aumento do diâmetro ocorreu mais rápido nos frutos de plantas que foram submetidas ao raleio, em ambos os estádios avaliados. Isso pode ser conferido ao menor número de frutos nas plantas pulverizadas com a maior concentração de ethephon, reduzindo a competição entre os drenos.

Com relação à última avaliação dos frutos, observou-se crescimento linear para a massa fresca e para os diâmetros, transversal e longitudinal, com o aumento das concentrações de ethephon (Figuras 5A e 5B). Em média, houve acréscimo de $66,3 \%$ na massa fresca dos frutos, nas plantas pulverizadas com a maior concentração de ethephon, em relação à dos frutos das plantas que não foram submetidas ao raleio químico. Em comparação ao tratamento sem raleio, observaram-se aumentos de 26,1 \% e 27,2\% nos diâmetros transversal e longitudinal, com a aplicação de $600 \mathrm{mg} \mathrm{L}^{-1} \mathrm{de}$ ethephon, respectivamente.

O aumento observado foi superior ao observado por Serciloto et al. (2003), em relação ao incremento no tamanho dos frutos, de 6,8\% em tangor 'Murcott', com a aplicação de ethephon para promover o raleio. Essa superioridade se deve às condições de baixa precipitação (Figura 1) durante o crescimento dos frutos, que limitou seu desenvolvimento, principalmente nas plantas que não foram submetidas ao raleio, cujos frutos ficaram sob maior competição. 


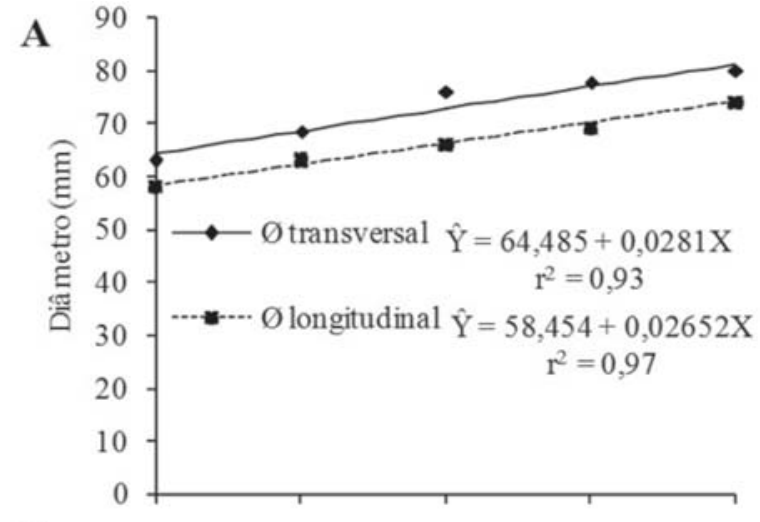

B

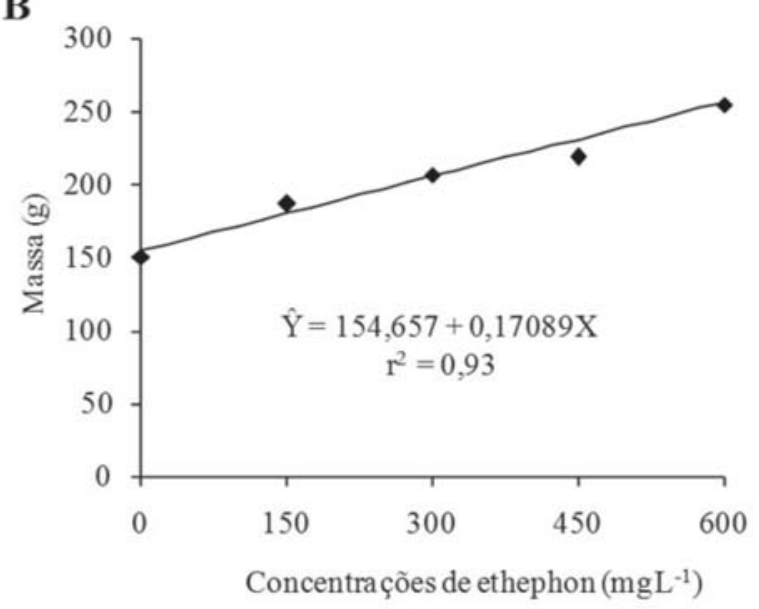

Figura 5. Diâmetro, transversal e longitudinal (A) e massa fresca (B) avaliados em frutos de tangerineira 'Ponkan' (Citrus reticulata Blanco), em função das concentrações de ethephon, no momento da colheita.

\section{CONCLUSÕES}

O raleio químico com a aplicação de ethephon favorece a obtenção de tangerinas ‘Ponkan’ com maior tamanho.

As concentrações a partir de $300 \mathrm{mg} \mathrm{L}^{-1}$ de ethephon são as mais adequadas para promover o raleio da tangerina 'Ponkan', nas condições estudadas.

O raleio realizado quando os frutos apresentam estádio de $40 \mathrm{~mm}$ proporciona a obtenção de frutos com os maiores diâmetros.

\section{AGRADECIMENTOS}

Ao Conselho Nacional de Desenvolvimento Científico e Tecnológico - CNPq, pela concessão de bolsa. Ao Professor Telde Natel Custódio, pela colaboração nas análises estatísticas.

\section{REFERÊNCIAS}

Agustí M, Almela V, Zaragoza S, Juan M, Trenor I, Alonso E \& Primo-Millo E (1998) Técnicas para mejorar el tamaño del fruto de naranjas y mandarinas. Cuadernos de Tecnologia Agrária, 3:1-15.
Agustí J, Zapater M, Iglesias DJ, Cercos, M, Tadeo, FR \& Talon M (2007) Differential expression of putative 9-cisepoxycarotenoid dioxygenases and abscisic acid accumulation in water-stressed vegetative and reproductive tissues of citrus. Plant Science, 172:85-94.

Bain JM (1958) Morphological, anatomical and physiological changes in the developing fruit of the Valencia orange, Citrus sinensis (L.) Osbeck. Australian Journal of Botany, 6:2-28.

Cruz MCM, Ramos JD, Lima LCO, Moreira RA \& Ramos PS (2009) Qualidade de frutas de tangerineira 'Ponkan' submetidas ao raleio químico. Revista Brasileira de Fruticultura, 31:127134.

Empresa Brasileira de Pesquisa Agropecuária - EMBRAPA (2006) Sistema brasileiro de classificação de solos. 2.ed. Rio de Janeiro, Centro Nacional de Pesquisa de Solos. 306p.

García-Luis A, Oliveira MEM, Bordón Y, Siqueira DL, Tominaga S \& Guardiola JL (2002) Dry matter accumulation in citrus fruit is not limited by transport capacity of the pedicel. Annals of Botany, 90:755-764.

Guardiola JL \& García-Luis A (2000) Increase size in citrus. Thinning and stimulation of fruit growth. Plant Growth Regulation, 31:121-132.

Mehouachi J, Serna D, Zaragoza S, Agustí M, Talon M \& Primo Millo E (1995) Desfoliation increases fruit abscission and reduces carbohydrate leaves in developing fruits and woody tissues of Citrus unshiu. Plant Science, 107:189-197.

Minchin PEH, Thorpe MR, Wunsche JN, Palmer JW \& Picton RF (1997) Carbon partitioning between apple fruits: short- and long-term response to availability of photosynthate. Journal of Experimental Botany, 48:1401-1406.

Ortolá AG, Monerri C, Guardiola JL, Garcia-Martinez JL \& Quinlan JD (1998) Fruitlet age and inflorescence characteristics affect the thinning and the increase in fruitlet growth rate induced by auxin applications in citrus. Acta Horticulturae, 463:501-508.

Ruiz R, García-Luis A, Monerri C \& Guardiola JL (2001) Carbohydrate availability in relation to fruitlet abscission in Citrus. Annals of Botany, 87:805-812.

Ruiz R \& Guardiola JL (1994) Carbohydrate and mineral nutrition of orange fruitlets in relation to growth and abscission. Physiologia Plantarum, 90:27-36.

Sanches FR (2000) Aplicação de biorreguladores vegetais: aspectos fisiológicos e aplicações práticas na citricultura mundial. Jaboticabal, Funep. 160p.

Serciloto CM, Castro PRC, Tavares S \& Medina CL (2003) Desbaste e desenvolvimento do tangor 'Murcott' com o uso de biorreguladores. Laranja, 24:65-68.

Spiegel-Roy P \& Goldschmidt EE (1996) Reproductive physiology: flowering and fruiting. In: Spiegel-Roy P \& Goldschmidt EE. Biology of citrus. Cambridge, Cambridge University Press. p.70125.

Talón M, Hedden P \& Primo-Millo E (1990) Gibberellins in Citrus sinensis: a comparison between seeded and seedless varieties. Journal of Plant Growth Regulation, 9:201-206.

Zaragoza S, Trenor I, Alonso E, Primo-Millo E \& Agustí M (1992) Treatments to increase the final fruit size on Satsuma Clausellina. Proceedings of the International Society of Citriculture, 2:725728.

Rev. Ceres, Viçosa, v. 57, n.4, p. 500-505, jul/ago, 2010 